Patiño, R., Rueda, F. y Díaz, I. (2020), Consolidación de estados financieros en el sector público en Colombia, conveniencia de realización en el caso del Distrito Capital. Contaduría Universidad de Antioquia, 76, 135-158 Doi: https://doi.org/10.17533/udea.rc.n76a07

\title{
Consolidación de estados financieros en el sector público en Colombia, conveniencia de realización en el caso del Distrito Capital
}

\author{
Ruth Alejandra Patiño Jacinto \\ rapatinoj@unal.edu.co \\ Universidad Nacional de Colombia \\ orcid: 0000-0001-6017-7666 \\ Felipe Rueda Céspedes \\ felipe.rueda@usantotomas.edu.co \\ Universidad Santo Tomás \\ orcid: 0000-0003-0150-2603
Irma Consuelo Díaz García Q.E.P.D. ${ }^{* *}$ idiaz@shd.gov.co Secretaría Distrital de Hacienda orcid: 0000-0001-7603-7377

* Artículo de investigación, realizado dentro del proyecto de investigación "Efectos del concepto de valor razonable en las entidades públicas en Colombia” ejecutado en el año 2018.

** Dedicado a Irma, mujer excepcional en todos los sentidos, gran ser humano, quien proporcionó importantes aportes a la construcción de este documento, compartiendo sus conocimientos y experiencia de una manera generosa. 
Consolidación de estados financieros en el sector público en Colombia, conveniencia de realización en el caso del Distrito Capital

Resumen: Una vez revisada la regulación contable pública en Colombia, se han evidenciado complicaciones en temas de consolidación puesto que se generaron tres marcos normativos, los cuales presentan divergencias entre ellos, asi pues, este trabajo pretende analizar cada uno de ellos, con el fin de conocer cuáles son las diferencias y así determinar la conveniencia de realizar la consolidación de estados financieros en el sector público en el Distrito Capital. Se utiliza una metodología cualitativa con el método de estudio de caso, con herramientas de análisis documental y una encuesta aplicada a las empresas objeto de consolidación de la entidad pública. Se determinó que las diferencias de los tratamientos contables utilizados por las empresas no generan gran complejidad para homogeneizar la información objeto de consolidación y por ende es oportuno realizarla.

Palabras clave: Entidades públicas, Normas Internacionales de Contabilidad, consolidación, contabilidad, estados financieros.

Financial statements consolidation in the Colombian public sector: convenience of carrying it out in the case of District Capital

Abstract: After reviewing public accounting regulation in Colombia, complications in consolidation matters have been evidenced since three regulation frameworks were generated, which diverge among them. Hence, this work seeks to analyze each one of them in order to know the differences and thus determine the convenience of carrying out the consolidation of financial statements in the public sector of the District Capital. A qualitative methodology is used with the case study method, with documentary analysis tools and a survey applied to the companies that are the object of consolidation of the public entity. It was determined that the differences in the accounting treatments used by the companies do not generate great complexity to homogenize the information that is consolidated and thus it is advisable to carry out its consolidation.

Keywords: Public entities, international accounting regulations, consolidation, accounting, financial statements.

Consolidação de estados financeiros no setor público na Colômbia, conveniência de realização no caso do Distrito Capital

Resumo: Uma vez que a regulação contábil pública na Colômbia foi revisada, tem se evidenciado complicações em assuntos de consolidação, já que foram gerados três marcos normativos, os quais apresentam divergências entre eles; então, este trabalho pretende analisar cada um deles, com o objetivo de conhecer quais são as diferenças e assim determinar a conveniência de realizar a consolidação de estados financeiros no setor público no Distrito Capital. Se utiliza uma metodologia qualitativa com o método de estudo de caso, com ferramentas de análise documental e uma enquete aplicada às empresas objeto de consolidação da entidade pública. Determinou-se que as diferenças dos tratamentos contábeis utilizados pelas empresas não geram grande complexidade para homogeneizar a informação objeto de consolidação e por tanto é oportuno realiza-la.

Palavras chave: Entidades públicas, Normas Internacionais de Contabilidade, consolidação, contabilidade, estados financeiros.

La consolidation des états financiers dans le secteur public en Colombie, intérêt de réalisation dans le cas du District Capital

Résumé : après avoir révisé la régulation comptable publique en Colombie, des complications liées à la consolidation ont été mise en évidence dues à la création de trois cadres normatifs, qui présentent des divergences parmi eux. Cet article vise à analyser chacun d'entre eux afin de connaître leur différences et de décider sur l'intérêt de réaliser la consolidation des état financiers dans le secteur public dans le District Capital colombien. D'abord, une methodologie qualitative avec la méthode d'étude de cas a été mise en place, accompagnée des outils d'analyse documentaire et d'un sondage fait aux entreprises objet de la consolidation de l'entité publique. Il a été décidé ensuite que les différences dans les traitements comptables mis en place par les entreprises ne sont pas d'une grande complexité pour homogéneiser l'information objet de consolidation et il convient donc de le faire.

Mots clés : entités publiques, normes internationales de comptabilité, consolidation, comptabilité, état financier. 


\title{
Consolidación de estados financieros en el sector público en Colombia, conveniencia de realización en el caso del Distrito Capital
}

\author{
Ruth Alejandra Patiño Jacinto, Felipe Rueda Céspedes, Irma Consuelo Díaz García ${ }^{\dagger}$ \\ https://doi.org/10.17533/udea.rc.n76a07
}

Primera versión recibida en febrero de 2020 - Versión aceptada en marzo de 2020

\section{Introducción}

\begin{abstract}
A partir de la Constitución Política de Colombia (Congreso de la República, 1991) se reivindica la importancia de la contabilidad del sector público, debido a que se comenzó a organizar el manejo de la contabilidad, puesto que se creó el cargo de Contador General de la Nación y La Contaduría General de la Nación [CGN] con funciones que llevarán a tener una organización contable del país. En este sentido se determina la creación del cargo de Contador General de la Nación, "quien llevará la contabilidad general de la nación y consolidará esta con la de sus entidades descentralizadas territorialmente o por servicio" (Art.354); así pues, debe ser el responsable de unificar y llevar a cabo la consolidación contable del sector público y por ende el Balance General de la Nación.

Luego de este suceso, la Ley 298 de 1996 trajo consigo la creación de la Contaduría General de la Nación al señalarla como "una Unidad Administrativa Especial, adscrita al Ministerio de Hacienda y Crédito Público, con personería jurídica, que está a cargo del Contador General de la Nación” (Congreso de la República, 1996, Artículo 1), con funciones de llevar la contabilidad general de la nación y determinar las políticas, principios y normas sobre contabilidad para el sector público (Presidencia de la República, 2004).

Al ser emitida la Resolución No. 354 de 2007 que "adopta el Régimen de Contabilidad Pública, se establece su conformación y se define el ámbito de aplicación” (Contaduría General de la Nación, 2007), se comenzó a entrar en un panorama internacional en el cual las normas que estaban mundialmente
\end{abstract}


Patiño, R., Rueda, F. y Díaz, I. Consolidación de estados financieros en el sector público en Colombia...

aceptadas para el sector público eran las emitidas por el IFAC ${ }^{1}$ denominadas IPSAS $^{2}$, lo que la CGN posteriormente toma como punto de partida para las entidades de gobierno.

Con la entrada en vigencia de la Ley 1314 de 2009, se determinaron las autoridades que deberían ser reguladoras y normalizadoras técnicas; se indicó que: "las facultades regulatorias en materia de contabilidad pública a cargo de la Contaduría General de la Nación, los Ministerios de Hacienda y Crédito Público y de Comercio, Industria y Turismo, obrando conjuntamente" (Congreso de la República, 2009, Artículo 6).

Una vez determinadas las entidades responsables para el proceso de adaptación de las Normas Internacionales, se comenzó a trabajar a partir del 2010 para emitir la regulación contable del sector público; de esta manera se decidió que era necesario agrupar a las entidades en tres tipos, por lo cual tendrían también tres marcos normativos diferentes tal y como se evidencia en la Figura No. 1.

Figura No. 1: Normativa Contable de las Entidades Públicas.

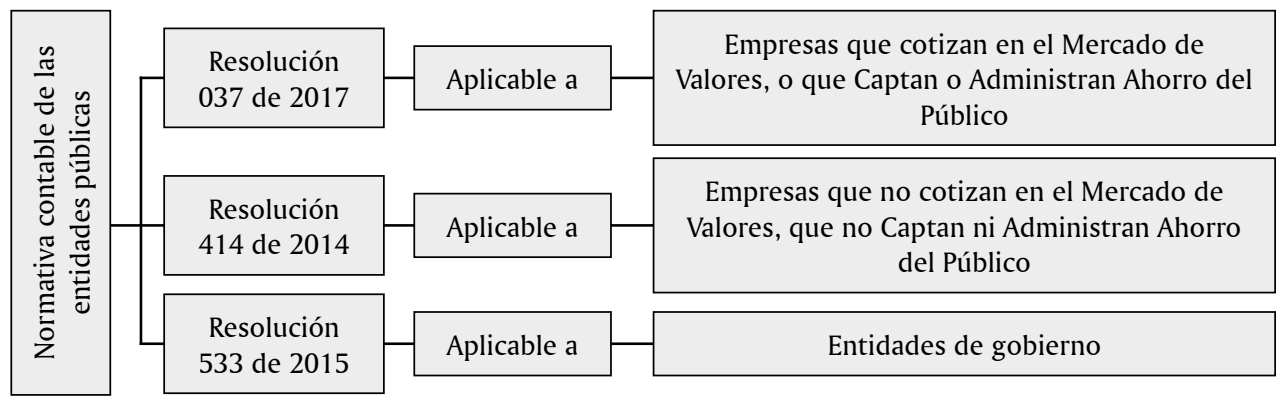

Fuente: Elaboración propia a partir de (Contaduría General de la Nación, sf).

En este proceso se determinó que las Entidades de Gobierno utilizan un marco normativo en convergencia con las Normas Internacionales del Sector Público NICSP, las empresas cotizantes en el mercado de valores, o que captan o administran ahorro del público aplican las Normas Internacionales de

1 International Federation of Accountants, “es una organización dedicada a servir al interés público por medio del fortalecimiento de la profesión contable y la contribución al desarrollo de fuertes economías internacionales. Está compuesta por 175 miembros y asociados en más de 130 países” (International Federation of Accountants, 2017).

2 The International Public Sector Accounting Standards Board, desarrolla normas contables y guías para uso de las entidades del sector público. "El IPSASB recibe el apoyo del Banco Mundial, el Banco Asiático de Desarrollo, los Contadores Profesionales Certificados de Canadá, el Consejo Sudafricano de Normas Contables, y los gobiernos de Canadá, Nueva Zelanda y Suiza.” (International Federation of Accountants, 2015). Las estructuras y los procesos que soportan las operaciones del IPSASB son facilitados por la IFAC. 
Información Financiera NIIF y las empresas que no cotizan en el mercado de valores, y que no captan ni administran ahorro del público tienen un modelo adaptado de las NIIF, esto de acuerdo con las características de cada tipo de entidad.

Dentro del ámbito del Sistema de Contabilidad Pública, un tema relevante es la Consolidación de Estados Financieros ya que genera información por un grupo de entidades, mostrando estos estados como si se tratara de un solo ente; esta información es pertinente para cumplir con los objetivos de la contabilidad pública "toma de decisiones, control y rendición de cuentas" (Contaduría General de la Nación, 2017a).

La consolidación de estados financieros se utiliza para recopilar y sistematizar los estados financieros de una matriz o controladora con sus subsidiarias o controladas, con el fin de contar con información completa y no parcial ya que corresponden a un grupo de entidades que son del mismo grupo económico y son útiles para tomar decisiones de tipo económico, especialmente de inversión; sin embargo, esto en contabilidad pública no tiene la misma lógica ya que los recursos son de la sociedad, de tal forma que la información sirve para tomar decisiones, ya no de inversión, y además como medio de control y rendición de cuentas.

El proceso de convergencia en normatividad contable pública en general ha traído diferencias en el reconocimiento de partidas y una diversidad de tratamiento de acuerdo a los referentes utilizados; esto conlleva a problemas en el proceso específico de consolidación de estados financieros, ya que para la agregación de la información se presentan inconsistencias si las partidas tienen criterios de reconocimiento, medición o presentación distintos; adicionalmente la consolidación para los estados financieros de entidades de gobierno es voluntaria, tomando en cuenta que las entidades deben considerar el costo-beneficio del proceso y decidir si es conveniente realizarlo; por ende, es relevante evaluar las implicaciones que tiene la realización de este procedimiento para casos específicos.

Es así como esta investigación surge con el propósito de dar solución al complejo tratamiento que se tiene para consolidar el sector público colombiano una vez realizada la convergencia contable a estándares internacionales, esto como consecuencia de la aplicación de tres marcos normativos diferentes que se generaron para cada tipo de entidad como se muestra en la Figura No. 1; por esto, se hace necesario conocer las diferencias que se tienen en temas de métodos de medición de algunas partidas con el fin de llevar a cabo el proceso de consolidación (International Federation of Accountants, 2017) de la información financiera pública, pues la información consolidada sirve para "determinar la verdadera dimensión de las administraciones públicas y para 
Patiño, R., Rueda, F. y Díaz, I. Consolidación de estados financieros en el sector público en Colombia...

conseguir la comparabilidad de la información contable de distintas entidades públicas" (Bravo, 2008, p. 135).

El análisis se hace en un caso muy representativo para el sector público que es el Distrito Capital, el cual por su magnitud agrupa entidades de gobierno, empresas que cotizan en bolsa y empresas que no cotizan en bolsa de valores.

El artículo incluye la revisión de la literatura, la metodología para la realización del estudio de caso, posteriormente se presenta la importancia y el proceso de la consolidación de los estados financieros, posteriormente se analizan las diferencias entre los tres marcos normativos de las entidades públicas, y finalmente, se establece el impacto de las diferencias en las empresas para el caso estudiado.

\section{Revisión de la literatura}

Desde la perspectiva académica se han venido desarrollando estudios correspondientes a los procesos de consolidación de estados financieros para las entidades públicas, concluyendo que está vigente la necesidad de investigar cuestiones técnicas y metodológicas, ya que esta información proporciona estadísticas macroeconómicas sobre las cuales se toman decisiones muy relevantes, además de la ausencia de sistematización de conocimiento sobre el tema (Santis y Grossi, 2018), así como de realizar un análisis empírico para generar regulación (Benito, Bastida y García, 2007). Lo anterior, evidencia la importancia del tema con distintos objetivos, ya que la información pública consolidada representa la agregación de grupos de entidades que comparten recursos públicos.

Por otra parte, un tema de discusión corresponde al objetivo central de la consolidación de estados financieros en el sector público, concluyendo que el fin corresponde a "determinar la verdadera dimensión de las administraciones públicas" (Bravo, 2008, p. 232) y facilitar la rendición de cuentas, lo cual es distante a lo que proponen los conjuntos de normas contables internacionales que se basan en la información útil para la toma de decisiones, inclusive en el conjunto normativo destinado para entidades públicas se menciona como objetivo central. De tal forma que un aspecto cuestionado es el traslado de normas y por tanto de conceptos y procedimientos del sector privado al sector público, cuyos procesos contables deben considerar las características particulares de las entidades públicas (Cirstea, Nistor y Tiron, 2017; Grossi y Steccolini, 2015; Lombarno, 2013).

En este mismo sentido, se plantea la necesidad de aplicar técnicas propias y no imitar las privadas como los parámetros planteados por las NIIF (Normas Internacionales de Información Financiera), modelo en el cual se eliminó el Método Proporcional de Consolidación (Lombarno, 2013), que incorporaba las partidas de acuerdo con la participación de la controladora, por lo que podría 
ser una alternativa para las entidades públicas, pero dado que no está vigente no es posible aplicarlo actualmente.

Otro aspecto objeto de análisis es la manera como se determina el control, característica que implica la necesidad de consolidar estados financieros; esto lo determina el área de consolidación, es decir, cuáles entidades se incorporan y cuáles no, dependiendo si cumplen con dicha característica. Dado lo anterior en necesario considerar las diferentes perspectivas desarrolladas respecto al control.

Referente a la determinación del control, este corresponde a la mayoría de los derechos de voto, lo que tiene relación con la propiedad; sin embargo, posteriormente se centró en la visión de poder de decisión y en los beneficios asociados a esta capacidad de decisión (Benito, Bastida y García, 2007); de esta determinación de control depende el área de consolidación desde las NICSP (Normas Internacionales de Sector Público), modelo sobre el cual se basa la regulación contable para las entidades de gobierno en Colombia; no obstante, una diferencia relevante es que en el país el proceso no es obligatorio, excepto el caso del consolidado general realizado por la CGN atendiendo sus competencias dadas en la Constitución Nacional.

Existen dos casos de determinación del área de consolidación: el enfoque de control desde la capacidad de decisión dado por la NIIF $10^{3}$ y la NICSP $35^{4}$; por otra parte, se encuentra el enfoque de financiación; es decir, que el área de consolidación depende de las entidades a las cuales financia la controladora (Santis y Grossi, 2018). De acuerdo con la Contaduría General de la Nación (2017c), el criterio para definir el control es una mezcla de las dos alternativas anteriores ya que consideran elementos de poder de decisión y también la dependencia económica, lo que Bisogno, Santis y Tommasetti (2015) mencionan como visión basada en el presupuesto.

Todo lo anterior es evidencia de que la información consolidada debe ser relevante y confiable (Cirstea, Nistor y Tiron, 2017); para cumplir diferentes objetivos; por último, sirven para obtener la imagen completa de nuevas formas de asociación como las Alianzas Público Privadas (APP), que necesitan información financiera para el control de recursos de estas actividades que son relevantes como el caso de la infraestructura (Santis y Grossi, 2018).

Diversos autores trabajan el tema desde la perspectiva de las restricciones que tiene la información consolidada para el sector público; se evidencia que no es reciente el debate de la conveniencia de informar un total de los recursos

3 Norma Internacional de Información Financiera 10 Estados Financieros Consolidados, aplicable a grandes empresas que tienen subsidiarias en el sector privado.

4 Norma Internacional de Contabilidad del Sector Público 35 Estados Financieros Consolidados, aplicable a entidades públicas controladas en algunos países. Colombia a través de la CGN decidió no aplicarla, ya que la Consolidación de Estados Financieros en este tipo de entidades es voluntaria. 
Patiño, R., Rueda, F. y Díaz, I. Consolidación de estados financieros en el sector público en Colombia...

públicos a partir de 1999; la discusión se centra en consolidar como si se tratara de una misma actividad, desconociendo que no se manejan las mismas bases contables y el hecho de que muchos de los recursos están restringidos y esto no es evidente en los reportes (Kinnersley, 2016); por otra parte, se cuestiona la medición a costo histórico, dado que en las normas contables internacionales del sector privado requieren la valoración a valor razonable (Gardini y Grossi, 2014); sin embargo, esta alternativa no se ha considerado en la normatividad colombiana.

Se identifica adicionalmente que la utilización del modelo de las NICSP presenta más costos que beneficios, dado que las normas son más cercanas a las normas privadas que al contexto público y el uso de la información generada es limitado, aunque, por otra parte, se evidencia que sirve para controlar la deuda y los gastos de un grupo de entidades públicas (Gomes, Brusca y Fernandes, 2019).

Por último, en lo concerniente a temas de análisis muy cercanos al del presente artículo, Jurado (2017), concluyó en su investigación que:

...existen temas que requieren ajustes a nivel del proceso de consolidación de la información que permiten cumplir a cabalidad con la definición de consolidación contable: Uniformidad en las políticas contables, eliminación de la totalidad de las transacciones recíprocas, revelación de la participación no controladora. (p. 106)

\section{Metodología}

La metodología que se utilizó es de carácter cualitativo, debido a que se analizaron componentes de los marcos normativos, así como las diferencias entre dichos marcos, con el fin de establecer la conveniencia de llevar a cabo el proceso de consolidación de estados financieros. Tuvo un enfoque de tipo descriptivo debido a que "consiste en la organización de información en forma útil y comprensible vía indicadores que faciliten la interpretación del fenómeno; los análisis multivariados permiten describir a las estructuras más complejas" (Ramírez y Zwerg, 2012, p. 107).

El análisis documental de las resoluciones por grupo de entidades se realizó por cada tema, identificando cuáles presentaban diferencias importantes en el reconocimiento, medición o presentación para los 3 tipos de entidades pública, lo que pudiera presentar efectos en el proceso de consolidación es estados financieros específicamente para el caso del Distrito Capital.

El método es el estudio de caso, ya que es un examen detallado del objeto de estudio (Katayama, 2014), el cual corresponde al proceso de consolidación de entidades y empresas públicas en Colombia; en específico, en el caso del Distrito Capital, la idea es lograr un análisis de la lógica y del procedimiento de consolidación y establecer la conveniencia de la realización para el caso analizado. 
La importancia de la consolidación del Distrito Capital se basa en que los organismos que financian los exigen para evaluar las condiciones financieras; por ello se analiza e identifica las restricciones que se pueden presentar en el proceso de elaboración de los estados financieros consolidados.

Los estados financieros analizados corresponden al sector público Distrito Capital, por lo cual incorporan entidades de gobierno y empresas; esto precisamente corresponde al problema de investigación, ya que siendo entidades de distinta naturaleza se presentan diferencias contables (3 Marcos Normativos) y por ende complejidades en la consolidación; situación que pretende analizarse para identificar si el beneficio de la información obtenida es mayor al costo y restricciones de presentarla.

Como instrumentos de investigación se apoyó en la investigación documental, puesto que esta investigación "busca recopilar la información contenida en documentos que son organizados sistemáticamente, descritos e interpretados" (Álvarez, 2003, p.108); los documentos académicos, los marcos normativos y documentos institucionales de la entidad que se estudió, aportaron las bases para identificar las ventajas y desventajas específicas para el Distrito Capital.

Otro instrumento utilizado fue una encuesta a empresas objeto de consolidación (Anexo N.1), acerca del reconocimiento de los elementos de los estados financieros bajo el nuevo marco normativo, con el fin de identificar los procedimientos realizados, y de esta forma determinar si es oportuno y aplicable realizar la consolidación de estados financieros; es decir, con el objetivo de analizar una de las dificultades que corresponde a las diferencias en tratamientos contables.

Dado que el área de consolidación incluye 6 empresas que cotizan en el mercado de valores, o que captan o administran ahorro del público, 14 empresas que no cotizan en el mercado de valores, y que no captan ni administran ahorro del público y 67 entidades de gobierno, se optó por identificar las diferencias presentadas en los marcos normativos de las empresas frente a las entidades de gobierno, de tal forma que la encuesta se aplicó en las 20 empresas, a la persona encargada de la contabilidad en cada una de ellas.

\section{Resultados}

\section{IV.I. Oportunidad de la consolidación en los estados financieros en} entidades de gobierno.

De acuerdo con la Contaduría General de la Nación CGN (2017c), las entidades de gobierno no están obligadas a consolidar los estados financieros con sus subsidiarias, sin embargo, si se garantiza la utilidad de los mismos, se 
Patiño, R., Rueda, F. y Díaz, I. Consolidación de estados financieros en el sector público en Colombia...

podrá realizar el proceso incluyendo a todas las subsidiarias; es decir, todas las entidades y empresas cotizantes o no, sobre las que se posea control.

El control parte de los beneficios, los riesgos y la capacidad de modificar la forma como se perciben (Contaduría General de la Nación, 2017c); esto quiere decir que la perspectiva de control surge de lo económico y de dirección, no necesariamente se debe tener participación mayoritaria ya que existen otros indicios como el control administrativo y la dependencia económica (Contaduría General de la Nación, 2017c), características específicas para el contexto de las entidades públicas en Colombia.

De esta manera se debe evaluar el costo - beneficio del proceso de consolidación de estados financieros en entidades de gobierno con el fin de establecer la oportunidad de llevar a cabo el proceso.

En primera medida la utilidad de la información está dada por los usuarios que realmente utilizarían estos estados financieros consolidados para tomar decisiones o para realizar algún tipo de control; por lo cual es relevante considerar el ¿para qué? y ¿para quién? se consolida con el fin de determinar si se justifica asumir los costos que implica el proceso.

Dentro de los costos se encuentran los asociados con determinar el control, lo cual puede tener diferentes niveles de complejidad; así como puede ser fácil de establecer puede ser muy complejo y costoso; por otra parte, en lo correspondiente a lo operativo, el software necesario para llevar a cabo el proceso, la capacitación del personal, entre otros; la determinación de las partidas recíprocas o inter-entidades, a fin de realizar las eliminaciones necesarias y no tener distorsiones en la información; y por último, un aspecto muy relevante, la necesidad de tener políticas contables homogéneas, lo cual es obligatorio en la regulación actual e implica procesos en marcos normativos distintos entre las entidades y empresas a consolidar.

En el caso estudiado se evaluaron los costos (Grossi, 2009) y los beneficios; de esta forma se determinó que la definición del control, los aspectos operativos como el software y la identificación de partidas específicas no tenían dificultades porque la entidad venía consolidando bajo el marco normativo contable previo.

Se definió que el beneficio para el caso de estudio es importante por cuanto en el marco de las competencias de la Secretaría Distrital de Hacienda, permite la generación de información por un grupo de entidades, como si se tratara de una sola entidad, para cumplir con los objetivos del Sistema de Contabilidad Pública, que las calificadoras de riesgos efectúen los análisis correspondientes al riesgo crediticio, que instituciones como el DANE analicen los aspectos macroeconómicos; internamente a nivel del gerente público, para analizar temas como el pasivo pensional, la deuda, entre otros, a cargo de diferentes entidades del sector central y descentralizado en Bogotá D.C. y establecer 
los temas técnico-contables que requieren mejora continua y así brindar la asesoría, documentación y capacitación técnica contable requerida.

Los estados contables consolidados reflejan la manera como el gobierno central influye en las decisiones de las demás entidades de gobierno y empresas y la retribución que se obtiene por la forma como se realiza dicho control.

Por último, son la información consolidada es útil como instrumento de control de recursos públicos para la sociedad. Entonces bajo las nuevas condiciones un factor importante para determinar la conveniencia de realizar el proceso de consolidación de estados financieros corresponde a la determinación de las diferencias entre las políticas contables en las empresas objeto de consolidación en el caso específico.

\section{IV.II. Proceso de Consolidación de Estados Financieros}

La Contaduría General de la Nación define algunos conceptos importantes para llevar a cabo el proceso de consolidación, primero se define el concepto consolidación contable:

Es el proceso de transformación extracontable que, a partir del reporte de la información cuantitativa y cualitativa de las ECP, recibida a través del sistema CHIP presenta la situación financiera, económica, social y ambiental, así como de actividad de un grupo de entidades como si se tratara de un solo ente. (Contaduría General de la Nación, 2014a)

De igual forma, se menciona que este es un proceso secuencial, en el cual se conforma un único estado financiero del que se debe "Eliminar las operaciones reciprocas, para determinar y registrar los saldos por conciliar a que haya lugar, de igual forma se reconoce la participación de los terceros en el patrimonio quedando los estados financieros consolidados como definitivos" (Contaduría General de la Nación, 2018).

El segundo concepto que es necesario definir es el análisis de cobertura, en este "se debe realizar una revisión de las condiciones de la información contable para posteriormente realizar el debido proceso de consolidación." (Contaduría General de la Nación, 2014a)

Para la CGN (2017c) se exige que las políticas de las entidades del área de consolidación deben ser homogéneas, lo cual da consistencia a la información consolidada con el fin de que cumpla con los distintos objetivos propuestos para dicha entidad. Todo lo anterior es relevante en cuanto a lo procedimental; sin embargo, debe cuestionarse en qué ocasiones se debe consolidar y para qué se realiza el proceso.

El proceso de consolidación es relevante para obtener información para todo el grupo de entidades del Distrito Capital, para tomar decisiones y para los entes encargados de la financiación. Para ello incluye todas las entidades sobre las cuales tiene control Bogotá D.C. como ente Territorial: 
Patiño, R., Rueda, F. y Díaz, I. Consolidación de estados financieros en el sector público en Colombia...

- Empresas que cotizan en el Mercado de Valores, o que Captan o Administran Ahorro del Público: Seis (6).

- Empresas que no Cotizan en el Mercado de Valores, y que no Captan ni Administran Ahorro del Público: Catorce (14).

- Entidades de Gobierno: Sesenta y siete (67).

IV.III. Análisis de las diferencias entre los 3 marcos normativos contables públicos en Colombia aplicables al caso de estudio

De acuerdo con el problema planteado, el objetivo es identificar las diferencias que se pueden presentar en los tres marcos normativos y que generan impacto en los estados financieros consolidados, por lo cual se incluye el resumen de los temas que son distintos en los marcos normativos; posteriormente el análisis de las diferencias y, por último, el impacto que tienen dichas diferencias en las empresas encuestadas correspondientes al caso de estudio.

IV.III.I. Temas en los cuales se presenta diferente normatividad que aplican al Distrito

Se identifica que los temas aplicables para el consolidado del sector público distrital y sus correspondientes normas o capítulos son los que se presentan en la Tabla No. 1.

Tabla No. 1: Temas aplicables a la consolidación en el sector público distrital y su norma correspondiente.

\begin{tabular}{lccc}
\hline \multicolumn{1}{c}{ TEMAS } & $\begin{array}{c}\text { Empresas que cotizan } \\
\text { en el mercado de } \\
\text { valores, o que captan } \\
\text { o administran ahorro } \\
\text { del público }\end{array}$ & $\begin{array}{c}\text { Empresas que no cotizan en } \\
\text { el mercado de valores, y que } \\
\text { no captan ni administran } \\
\text { ahorro del público } \\
\text { (Resolución No. 414) de 2014 }\end{array}$ & $\begin{array}{c}\text { Entidades } \\
\text { de gobierno } \\
\text { (Resolución No. } \\
\text { 533 de 2015) }\end{array}$ \\
\hline Inventarios & NIC 2 & Capítulo I & Capítulo I \\
Propiedad, planta y equipo & NIC 16 & Capítulo I & Capítulo I \\
Propiedades de inversión & NIC 40 & Capítulo I & Capítulo I \\
Arrendamientos & NIC 17 - NIF $16(2020)$ & Capítulo I & Capítulo I \\
Intangibles & NIC 38 & Capítulo I & Capítulo I \\
Instrumentos Financieros & NIC 32 - NIC 39 - NIIF 9 & Capítulo I y II & Capítulo I y II \\
$\begin{array}{l}\text { Activos no corrientes } \\
\text { disponibles para la venta }\end{array}$ & NIIF 5 & & Capítulo I \\
Inversiones en Controladas & NIC 27 & Capítulo I & Capítulo I \\
Inversiones en Asociadas y & NIC 28 & Capítulo I y II & Capítulo I \\
Negocios Conjuntos & NIIF 11 &
\end{tabular}




\begin{tabular}{|c|c|c|c|}
\hline TEMAS & $\begin{array}{c}\text { Empresas que cotizan } \\
\text { en el mercado de } \\
\text { valores, o que captan } \\
\text { o administran ahorro } \\
\text { del público } \\
\end{array}$ & $\begin{array}{l}\text { Empresas que no cotizan en } \\
\text { el mercado de valores, y que } \\
\text { no captan ni administran } \\
\text { ahorro del público } \\
\text { (Resolución No. 414) de } 2014\end{array}$ & $\begin{array}{c}\text { Entidades } \\
\text { de gobierno } \\
\text { (Resolución No } \\
533 \text { de 2015) }\end{array}$ \\
\hline $\begin{array}{l}\text { Deterioro en el valor de los } \\
\text { activos }\end{array}$ & NIC 36 & Capítulo I & Capítulo I \\
\hline Ingresos & NIIF 15 & Capítulo IV & Capítulo IV \\
\hline $\begin{array}{l}\text { Beneficios a empleados y } \\
\text { planes por beneficios de } \\
\text { retiro }\end{array}$ & NIC 19, NIC 26 & Capítulo II & Capítulo II \\
\hline Subvenciones de gobierno & NIC 20 & Capítulo IV & \\
\hline Costos por préstamos & NIC 23 & Capítulo I & Capítulo I \\
\hline Impuesto a las ganancias & NIC 12 & Capítulo V & \\
\hline Provisiones & NIC 37 & Capítulo II & Capítulo II \\
\hline Combinaciones de Negocios & NIIF 3 & Capítulo VI & Capítulo V \\
\hline $\begin{array}{l}\text { Efectos de las Variaciones } \\
\text { en las Tasas de Cambio de la } \\
\text { Moneda Extranjera }\end{array}$ & NIC 21 & Capítulo V & Capítulo V \\
\hline
\end{tabular}

Fuente: Elaboración propia a partir de (Contaduría General de la Nación, 2017b), (2014b), (2017c).

A partir de esta primera revisión se identifica una serie de temas que deben ser analizados por cuanto tienen diferentes referentes normativos, estos son: inventarios, propiedades, planta y equipo, propiedades de inversión, arrendamientos, intangibles, instrumentos financieros, activos no corrientes disponibles para la venta (en este caso el inconveniente surge de la ausencia del tema en dos marcos normativos, por lo cual se pueden presentar diferentes interpretaciones), inversiones en controladas, inversiones en asociadas y negocios conjuntos, operaciones conjuntas, deterioro en el valor de los activos, ingresos, beneficios a empleados y planes por beneficios de retiro, subvenciones de gobierno, costos por préstamos, impuesto a las ganancias, provisiones, combinaciones de negocios y efectos de las variaciones en las tasas de cambio de la moneda extranjera.

\section{IV.III.II. Análisis de las diferencias que tienen impactos por cada tema}

Luego de identificar los temas en los cuales es posible tener diferencias se analizan cada uno de ellos y se determina que los potencialmente generadores de impactos en los estados financieros consolidados son:

IV.III.II.I. Propiedades, planta y equipo, propiedades de inversión y activos intangibles

Se encuentra que las diferencias en estos tres temas son las mismas: 
Patiño, R., Rueda, F. y Díaz, I. Consolidación de estados financieros en el sector público en Colombia...

- En el caso de las empresas cotizantes en el mercado de valores, o que captan o administran ahorro del público, tienen la posibilidad de seleccionar la política del costo revaluado ${ }^{5}$; en tal caso lo aplican para todos los elementos de una clase de propiedades, planta y equipo; si la empresa opta por esta alternativa generaría diferencias de valoración por cuanto los otros dos marcos normativos consideran el costo como única alternativa; es decir, unos tendrían esta partida por valor razonable y otros por costo.

- Para las entidades de gobierno la medición del deterioro es distinta en el caso de los activos no generadores de efectivo ${ }^{6}$; sin embargo, se determina que en el caso de estudio, la naturaleza de los activos corresponde a generadores de efectivo, por lo cual no se presentan diferencias de política.

\section{IV.III.II.II. Arrendamientos}

En el caso del arrendamiento financiero para el caso del arrendatario ${ }^{7}$, las empresas cotizantes reconocen el activo y el pasivo por el valor razonable del bien arrendado, mientras las empresas no cotizantes y las entidades de gobierno reconocen el activo con una medición de acuerdo con la naturaleza del activo. En caso de que las empresas y las entidades de gobierno actúen en el papel de arrendatario en arrendamientos financieros, generarían una diferencia importante en la valoración, por cuanto las empresas que cotizan en bolsa son las únicas que valoran estos bienes por el criterio de valor razonable.

IV.III.II.III. Instrumentos financieros

- El deterioro de cuentas por cobrar y préstamos por cobrar se estima por el modelo de pérdidas esperadas en el marco normativo de las empresas cotizantes en el mercado de valores, o que captan o administran ahorro del público, mientras que en el correspondiente a empresas no cotizantes y entidades de gobierno se calcula por el modelo de pérdidas incurridas. Esto implica unas diferencias importantes en la valoración por cuanto la lógica y por ende los resultados son distintos.

La situación es similar para el caso del deterioro de las inversiones de administración de liquidez por costo amortizado y el deterioro de las inversiones de administración de liquidez al valor razonable (valor de mercado) con cambio al ORI (patrimonio); se lleva por el modelo de pérdidas esperadas para empresas cotizantes, mientras para no cotizantes y entidades de gobierno se determina por el comparativo con valor de mercado.

5 Se menciona como valor razonable para las propiedades de inversión.

6 Para este caso se consideran los activos en los cuales los beneficios específicamente corresponden al potencial de servicio y no a beneficios económicos.

7 Se presenta en el arrendamiento financiero dada la transferencia de riesgos y beneficios. 
- En el marco normativo de las empresas que cotizan no se encuentra la categoría de inversiones al costo; sin embargo, se identifica que la naturaleza y las características de estos activos es distinta, por lo cual no genera impactos al considerarse activos distintos.

IV.III.II.IV. Activos no corrientes disponibles para la venta

En el caso de las empresas cotizantes la clasificación y medición es específica para este tipo de partidas; por el contrario, puede encontrarse en diferentes clases de activos en las empresas no cotizantes y entidades de gobierno, en caso de contar con ellos; si sucede este hecho generaría diferencias en presentación y medición de los activos.

IV.III.II.V. Inversiones en Controladas, Negocios Conjuntos y Asociadas

- En el concepto de control de las entidades de gobierno existe una diferencia correspondiente a los riesgos y dependencia económica; es decir, no son solo rendimientos, lo que genera situación de control como se plantea en los otros dos modelos. Sin embargo, partiendo de que en el caso de estudio la controladora es una sola, esto no genera impactos.

- Adicionalmente existe la posibilidad en marco normativo de empresas que cotizan de utilizar métodos distintos al método de participación patrimonial, como el costo o el valor razonable. En caso de optar por ello generan diferencias importantes en este tipo de inversiones. Esta diferencia es similar para inversiones en asociadas y en negocios conjuntos.

IV.III.II.VI. Ingresos

- Con la NIIF 15 (aplicable a empresas cotizantes) se presenta una diferencia, ya que en esta se evalúan más las condiciones contractuales para definir el tratamiento contable de los ingresos. Esto genera diferencias posteriores, por lo cual se debe considerar dar unos lineamientos para lograr homogeneidad en este aspecto.

- La segunda diferencia es la especificación de los ingresos sin contraprestación; aunque corresponden a hechos que se presentan solo en entidades de gobierno, por cuanto la naturaleza es distinta, esto no genera impacto.

IV.III.II.VII. Subvenciones de Gobierno

En el marco normativo de empresas no cotizantes se otorgan más alternativas de medición para subvenciones en activos distintos al efectivo, por lo que si las empresas presentan estas transacciones económicas se debe lograr hacer homogénea la medición de dichos activos. 
Patiño, R., Rueda, F. y Díaz, I. Consolidación de estados financieros en el sector público en Colombia...

\section{IV.III.II.VIII. Partidas que no presentan diferencias relevantes}

Luego del análisis se encuentra que las siguientes partidas no generan diferencias importantes en las políticas de los tres marcos normativos:

- Inventarios.

- Operaciones conjuntas.

- Beneficios a empleados y planes por beneficios de retiro.

- Costos por préstamos.

- Impuesto a las ganancias.

- Provisiones.

- Combinaciones de negocios.

- Efectos de las Variaciones en las Tasas de Cambio de la Moneda Extranjera. A continuación, se presenta un resumen de las principales diferencias encontradas:

Tabla No. 2: Temas en los cuales se identifican diferencias en el caso de estudio.

\begin{tabular}{|c|c|}
\hline TEMAS & Diferencia \\
\hline $\begin{array}{l}\text { Propiedad, planta y equipo, } \\
\text { Propiedades de inversión e } \\
\text { Intangibles }\end{array}$ & $\begin{array}{l}\text { Las empresas que cotizan en el mercado de valores, o que captan o } \\
\text { administran ahorro del público; Pueden optar por la política de } \\
\text { valoración del costo revaluado. }\end{array}$ \\
\hline $\begin{array}{l}\text { Arrendamientos financieros } \\
\text { para el caso de arrendatarios }\end{array}$ & $\begin{array}{l}\text { Las empresas que cotizan en el mercado de valores, o que captan o } \\
\text { administran ahorro del público reconocen el activo a valor razonable. }\end{array}$ \\
\hline Instrumentos Financieros & $\begin{array}{l}\text { Las empresas que cotizan en el mercado de valores, o que captan o } \\
\text { administran ahorro del público realizan la medición del deterioro de } \\
\text { las cuentas por cobrar, los préstamos por cobrar, las inversiones de } \\
\text { administración de liquidez por costo amortizado, y las inversiones de } \\
\text { administración de liquidez a valor de mercado con cambios al ORI por el } \\
\text { método de las pérdidas esperadas. }\end{array}$ \\
\hline $\begin{array}{l}\text { Inversiones en controladas, } \\
\text { asociadas y negocios } \\
\text { conjuntos }\end{array}$ & $\begin{array}{l}\text { Las empresas que cotizan en el mercado de valores, o que captan o } \\
\text { administran ahorro del público tienen la posibilidad de utilizar métodos } \\
\text { distintos al método de participación patrimonial, como el costo o el } \\
\text { valor razonable }\end{array}$ \\
\hline Ingresos & $\begin{array}{l}\text { Las empresas que cotizan en el mercado de valores, o que captan o } \\
\text { administran ahorro del público a partir de la vigencia de la NIIF } 15 \text { deben } \\
\text { reconocer los ingresos de acuerdo con cumplimiento de las obligaciones } \\
\text { de desempeño. }\end{array}$ \\
\hline $\begin{array}{l}\text { Activos no corrientes } \\
\text { disponibles para la venta }\end{array}$ & $\begin{array}{l}\text { Las empresas que cotizan en el mercado de valores, o que captan o } \\
\text { administran ahorro del público presentan un tratamiento específico } \\
\text { mientras para las empresas que no cotizan en el mercado de valores, } \\
\text { y que no captan ni administran ahorro del público y las entidades de } \\
\text { gobierno no esta determinado en el marco normativo correspondiente. }\end{array}$ \\
\hline Subvenciones de gobierno & $\begin{array}{l}\text { Para las empresas que no cotizan en el mercado de valores, y que no } \\
\text { captan ni administran ahorro del público se otorgan más alternativas de } \\
\text { medición para subvenciones en activos distintos al efectivo. }\end{array}$ \\
\hline
\end{tabular}

Fuente: Elaboración propia. 
Sobre los temas que presentan diferencias, se indaga la información disponible que tienen las empresas tanto cotizantes como no cotizantes, a fin de establecer qué tan complejo y costoso es aplicar, para efectos de consolidación, las mismas políticas por medio de ajustes en el momento del proceso de consolidar los estados financieros.

Se opta por indagar en las empresas ya que son menor en número que las entidades de gobierno, lo cual lleva a considerar que es más fácil ajustar el modelo de las empresas hacia el modelo de las entidades de gobierno y no lo contrario, con el fin de lograr políticas contables similares. Para esto se realiza la encuesta a las empresas en la siguiente fase del estudio.

IV.IV. Análisis de los impactos de las diferencias de los modelos contables en las empresas específicas del caso

Resultado de la encuesta los hallazgos son los siguientes:

IV.IV.I. Deterioro en las inversiones al costo amortizado e inversiones al valor razonable con cambio al ORI:

Ninguna de las empresas presenta deterioro de este tipo de inversiones; sin embargo, en el caso de presentarlas, las empresas cotizantes que aplican NIIF deberán calcular el deterioro no solo por las pérdidas esperadas, de acuerdo con su marco normativo, sino por el modelo de pérdidas incurridas y proporcionar la información con el fin de efectuar el correspondiente ajuste.

IV.IV.II. Deterioro en las cuentas por cobrar:

Tres empresas cotizantes presentan deterioro de esta cuenta, por lo que deberán calcular el deterioro no solo por las pérdidas esperadas, de acuerdo con su marco normativo, sino por el modelo de pérdidas incurridas y proporcionar la información con el fin de efectuar el correspondiente ajuste.

IV.IV.III. Aplicación del costo revaluado para propiedad, planta y equipo, propiedad de inversión o activos intangibles:

Una empresa aplica el costo revaluado para propiedad, planta y equipo; caso en el cual deberá identificar el costo de la partida y proporcionar la información con el fin de efectuar el correspondiente ajuste para efectos de la consolidación; la empresa cuenta con esta información.

IV.IV.IV. Arrendamiento financiero como arrendatario:

Una empresa cotizante que presenta este tipo de transacciones deberá identificar el costo del activo correspondiente, ya que lo reconoce por el valor razonable, y proporcionar la información con el fin de efectuar el correspondiente ajuste. La empresa menciona que puede obtener esta información fácilmente. 
Patiño, R., Rueda, F. y Díaz, I. Consolidación de estados financieros en el sector público en Colombia...

\section{IV.IV.V. Activos no corrientes disponibles para la venta:}

Una empresa cotizante o no cotizante que presente este tipo de activos deberá identificar el costo del activo correspondiente, ya que lo reconoce por el valor razonable, y proporcionar la información con el fin de efectuar el correspondiente ajuste; en el momento del estudio ninguna tiene.

IV.IV.VI. Subvenciones de activos no monetarios:

Una empresa no cotizante que presente este tipo de transacciones deberá identificar el costo o el valor del intercambio del activo correspondiente, y proporcionar la información con el fin de efectuar el ajuste. En el momento no hay casos de subvenciones que tengan esta naturaleza de no monetarios.

\section{Conclusiones}

La literatura académica muestra que se presentan diversas limitantes en la consolidación de entidades públicas; partiendo del análisis de un caso de una entidad de gobierno como controladora de empresas cotizantes y no cotizantes en mercados públicos de valores, y considerando que los tres tipos de entidades públicas en Colombia tienen marcos normativos distintos y que adicionalmente la consolidación de estados financieros en entidades de gobierno es un proceso voluntario, se procedió a analizar la oportunidad de realizarlo partiendo de la perspectiva de costo - beneficio.

Se analizó el beneficio del proceso concluyendo que lo principal es proporcionar información para suministrar a las entidades que otorgan financiación importante para los proyectos, no solo de la controladora sino del grupo en general; esto para la entidad se consolida; sin embargo, existe un beneficio social y es obtener información por el conjunto de entidades para realizar control sobre los recursos públicos. En lo relacionado con costos, se identificó que lo principal corresponde a la homologación de políticas, dado que existen diferencias entre los modelos, ya que temas como software y capacitación del personal para el caso del Distrito Capital no son relevante ya que están preparados y realizan este proceso.

Se lograron identificar estas diferencias entre los tres marcos normativos en partidas específicas de propiedades, planta y equipo, propiedades de inversión, activos intangibles, arrendamientos para arrendatarios, instrumentos financieros, activos no corrientes disponibles para la venta, inversiones en controladas, negocios conjuntos y asociadas, ingresos y subvenciones, y se estableció que el principal impacto se centra en los criterios de medición.

Para el caso de estudio las diferencias están en dos partidas: i) Propiedades, planta y equipo, pues una empresa del grupo de consolidación realiza la valoración de la partida por revaluado y no por costo; y ii) Deterioro de cartera, ítem en el cual tres empresas calcularon el deterioro a sus cuentas por cobrar 
aplicando la medición por pérdidas esperadas, en razón a que el marco de las empresas cotizantes en el mercado de valores, o que captan o administran ahorro del público que utiliza NIIF indica este procedimiento; mientras que las demás (empresas que no cotizan en el mercado de valores, y que no captan ni administran ahorro del público y las entidades de gobierno) utilizan el método de pérdidas incurridas. Para las diferencias de las partidas se indagó con las empresas y los responsables mencionaron que, dado que manejan NIIF, cuentan con la información necesaria para hacer homogéneas las políticas con la controladora sin realizar ningún tipo de proceso adicional. Lo anterior lleva a concluir que el proceso de consolidación en el estudio de caso no presenta costos excesivos; por el contrario, el beneficio es evidente y se basa en contar con información consolidada para presentar a entidades externas con el fin de lograr financiación.

\section{Referencias bibliográficas}

Álvarez, J. L. (2003). Cómo hacer investigación cualitativa. Ciudad de México: Paidós Educador. Benito, B., Bastida, F. y García, T. (2007). La consolidación de cuentas en las entidades locales españolas: un estudio empírico. Revista Española de Financiación y Contabilidad, 36(134), 351-388.

Bisogno, M., Santis, S. y Tommasetti, A. (2015). Public-Sector Consolidated Financial Statements: An Analysis of the Comment Letters on IPSASB's. Exposure Draft No. 49. International Journal of Public Administration, (38), 311-324.

Bravo, V. N. (2008). Líneas de reforma de la Contabilidad Pública en España. Presupuesto y Gasto Público, 51, 231-242.

Cirstea, A., Nistor, S. y Tiron, A. (2017). Consolidated financial statements - a new challenge for the public sector administration. Journal of Economic and Administrative Sciences, 33(1), 46-65.

Congreso de la República. (1991). Constitución Política de Colombia. Obtenido de http:// www.secretariasenado.gov.co/senado/basedoc/constitucion_politica_1991.html

Congreso de la República. (1996). Ley 298 de 1996. "Por la cual se desarrolla el artículo 354 de la Constitución Política, se crea la Contaduría General de la Nación como una Unidad Administrativa Especial Adscrita al Ministerio de Hacienda y Crédito Público, y se dictan otras disposiciones". Obtenido de http://www.suin.gov.co/viewDocument. asp?ruta $=$ Leyes $/ 1657792$

Congreso de la República. (2009). Ley 1314 de 2009. “Por la cual se regulan los principios y normas de contabilidad e información financiera y de aseguramiento de información aceptados en Colombia, se señalan las autoridades competentes, el procedimiento para su expedición y se determinan las entidades responsables de vigilar su cumplimiento".. Obtenido de http://suin.gov.co/viewDocument.asp?ruta=Leyes/1677255

Contaduría General de la Nación. (2007). Resolución 354. “Por la cual se adopta el Régimen de Contabilidad Pública, se establece su conformación y se define el ámbito de aplicación". Obtenido de https://mintic.gov.co/portal/604/articles-3790_documento. pdf 
Patiño, R., Rueda, F. y Díaz, I. Consolidación de estados financieros en el sector público en Colombia...

Contaduría General de la Nación. (2014a). Proceso de Consolidación Contable. 4. Bogotá, Colombia. Recuperado el 2018 de Octubre de 25, de http://www. contaduria.gov.co/wps/wcm/connect/cefc3632-d901-4f52-a44d-a2c5c105271b/ CONSOLIDACI\%C3\%93N+CONTABLE.pdf?MOD =AJPERES\&C

Contaduría General de la Nación. (2014b). Resolución 414 de 2014. "Por la cual se incorpora, en el Regímen de Contabilidad Pública, el marco normativo aplicable para algunas empresas sujetas a su ámbito y se dictan otras disposiciones”. Bogotá, Colombia.

Contaduría General de la Nación. (2017a). Referente Teórico y Metodológico de la Regulación Contable Pública. Obtenido de http://www.contaduria.gov.co/wps/wcm/ connect/682ee455-6e72-4dc2-b13a-77e726782739/Ref + te\%C3\%B3rico +y+metodo 1\%C3\%B3gico +\%2802-10-2017\%29.pdf?MOD =AJPERES\&CACHEID =682ee455-6e724dc2-b13a-77e726782739

Contaduría General de la Nación. (2017b). Resolución 037 del 2017. "Por la cual se regula el Marco Normativo para Empresas que Cotizan en el Mercado de Valores, o que Captan o Administran Ahorro del Publico”. Bogotá, Colombia.

Contaduría General de la Nación. (2017c). Resolución 484 de 2017 “Por la cual se modifican el anexo de la Resolución 533 de 2015 en lo relacionado con las Normas para el Reconocimiento, Medición, Revelación y Presentación de los Hechos Económicos del Marco Normativo para Entidades de Gobierno y e. Bogotá, Colombia.

Contaduría General de la Nación. (2018). Consolidación contable. Bogotá, Colombia.

Contaduría General de la Nación. (sf). RCP en convergencia con NIIF-NICSP. Obtenido de http:/www.contaduria.gov.co/wps/portal/internetes/home/internet/rcp1/rcp-niif/ marco-normativo-res-743/!ut/p/b1/04_Sj9CPykssy0xPLMnMz0vMAfGjzOINzPyDTEPdQoMDTV3NDBwdjQx9XEKNjU38DYEKIkEKcABHA1T9RqYGJkBBU0NLkyA_I4MAE5hHBYYmBBnvzHQWAMLPyAzUx8nSwPPkOCQQDdfVyM

Gardini, S. y Grossi, G. (2014). Voluntary adoption of the consolidated financial statement and fair value accounting by italian local governments. Journal of Public Budgeting, Accounting \& Financial Management, 26(2), 313-344.

Gomes, P., Brusca, I. y Fernandes, M. (2019). Implementing the International Public Sector Accounting Standards for consolidated financial statements: facilitators, benefits and challenges. Public Money \& Management, 39(8), 544-552.

Grossi, G. (2009). New development: Consolidated financial reporting as a stimulus for change in Italian local government. Public Money \& Management, 29(4), 261-264.

Grossi, G. y Steccolini, I. (2015). Pursuing Private or Public Accountability in the Public Sector? Applying IPSASs to Define the Reporting Entity in Municipal Consolidation. International Journal of Public Administration, 38, 325-334.

International Federation of Accountants. (2015). IFAC. Recuperado el 24 de Septiembre de 2017, de IFAC: https://www.ifac.org/news-events/2015-04/el-ipsasb-publica-directrizde-pr-ctica-recomendada-reporte-de-la-informaci-n-so

International Federation of Accountants. (2017). IFAC. Recuperado el 24 de Septiembre de 2017, de IFAC: https://www.ifac.org/about-ifac

Jurado, A. M. (2017). El proceso de consolidación de la información contable pública en Colombia: un análisis crítico y sus perspectivas de mejora. Bogotá D.C, Colombia. 
Katayama, R. (2014). Introducción a la investigación cualitativa: Fundamentos, métodos, estrategias y técnicas. Lima: Universidad Inca Garcilaso de la Vega.

Kinnersley, R. (2016). The development of the totals column on the combined balance sheet for state and local governments in the United States during the 20th century. The Accounting Historians Journal, 43(1), 33-57.

Lombarno, A. (2013). IPSAS and local government consolidated financial statementsproposal for a territorial consolidation method. Public Money \& Management, 33(6), 429-436.

Presidencia de la República. (2004). Decreto 143 de 2004. "Por el cual se modifica la estructura de la Contaduría General de la Nación y se determinan las funciones de sus dependencias". Obtenido de http://www.suin.gov.co/viewDocument. asp?ruta $=$ Decretos $/ 1032247$

Ramírez, F. H. y Zwerg, A. (2012). Metodología de la investigación: más que una receta. AD-minister, (20), 91-111.

Santis, S. y Grossi, G. (2018). Public sector consolidated financial statements: a structured literature review. Journal of Public Budgeting, Accounting \& Financial Management, 30(2), 230-251. 
Patiño, R., Rueda, F. y Díaz, I. Consolidación de estados financieros en el sector público en Colombia...

Anexo No. 1 - Principales preguntas aplicadas en la encuesta sobre reconocimiento de activos bajo el marco normativo contable para empresas con participación distrital

Responda las siguientes preguntas tomando en cuenta la situación en la aplicación del Nuevo Marco Normativo Contable.

1. ¿Las inversiones a costo amortizado presentan deterioro?

Sí $\square \quad$ NO $\square$

En caso de ser positiva la respuesta a la pregunta 1 responda las preguntas 2 y 3 .

2. ¿La empresa realiza medición del riesgo de crédito de las inversiones a costo amortizado?

Sí $\square \quad$ NO $\square$

3. Mencione detalladamente cómo realizan la medición del deterioro de las Inversiones a costo amortizado, tomando en cuenta todas las variables como: tasa de interés utilizada, plazos normales, indicios de deterioro, entre otros.

4. ¿Las inversiones a valor razonable con cambios al ORI presentan deterioro? Sí $\square \quad$ NO $\square$

En caso de ser positiva la respuesta a la pregunta 4 responda las preguntas 5 y 6 .

5. ¿La empresa realiza medición del riesgo de crédito de las inversiones a valor razonable con cambios al ORI?

Sí $\square \quad$ NO $\square$

6. Mencione detalladamente cómo realizan la medición del deterioro de las Inversiones a valor razonable con cambios al ORI, tomando en cuenta todas las variables como: tasa de interés utilizada, plazos normales, indicios de deterioro, entre otros.

7. ¿Las cuentas por cobrar de la empresa, presentan deterioro?

Sí $\square \quad$ NO $\square$

En caso de ser positiva la respuesta a la pregunta 7 responda las preguntas 8 y 9. 
8. ¿La empresa realiza medición del riesgo de crédito de las cuentas por cobrar?

Sí $\square \quad \mathrm{NO} \square$

9. Mencione detalladamente cómo la empresa realiza la medición del deterioro de cartera, tomando en cuenta todas las variables como: tasa de interés utilizada, plazos normales, indicios de deterioro, entre otros.

10. Especifique el criterio utilizado para valorar cada una de las siguientes partidas:

\begin{tabular}{lll}
\hline \multicolumn{1}{c}{ Partida } & Costo & $\begin{array}{c}\text { Revaluado } \\
\text { (Valor razonable) }\end{array}$ \\
\hline Propiedades, planta y equipo & \\
\hline Propiedad de inversión & \\
\hline Intangibles & \\
\hline
\end{tabular}

En caso de utilizar el criterio del revaluado en alguna de las tres partidas responda:

11. ¿La empresa tiene información separada del costo del activo y los correspondientes ajustes, así como la contrapartida de la aplicación de dichos ajustes que dieron lugar al valor "revaluado"?

\begin{tabular}{lll}
\hline Partida & Sí & NO \\
\hline Propiedades, planta y equipo & \\
\hline Propiedad de inversión & \\
\hline Intangibles
\end{tabular}

12. ¿La Empresa tiene bienes en arrendamiento financiero como arrendatario? Sí $\square \quad$ NO $\square$

Si su respuesta a la pregunta 12 es positiva, solicitamos remitir la información correspondiente a dichos arrendamientos: valor, duración, clasificación del activo, criterio de valoración del activo y el pasivo relacionado.

13. ¿La empresa tiene activos no corrientes disponibles para la venta?

Sí $\square \quad$ NO $\square$

En caso de ser positiva la respuesta a la pregunta 13 responda las preguntas 14, 15 y 16. 
Patiño, R., Rueda, F. y Díaz, I. Consolidación de estados financieros en el sector público en Colombia...

14. ¿Cuál es el valor de dichos activos?

15. ¿Cuál es el criterio de medición de dichos activos?

16. ¿En qué clase de activos se presentan?

17. ¿La empresa tiene inversiones en controladas, asociadas o negocios conjuntos?

Sí $\square \quad$ NO $\square$

En caso de ser positiva la respuesta a la pregunta 17 responda la pregunta 18 .

18. ¿Cuál es el criterio de reconocimiento de las Inversiones?

\begin{tabular}{lccc}
\hline \multicolumn{1}{c}{ Partida } & Costo & $\begin{array}{c}\text { Método de participación } \\
\text { patrimonial }\end{array}$ & $\begin{array}{c}\text { Valor } \\
\text { razonable }\end{array}$ \\
\hline Inversiones en controladas & & \\
\hline Inversiones en asociadas & & \\
\hline Inversiones en negocios conjuntos & & \\
\hline
\end{tabular}

19. ¿La empresa tiene subvenciones?

Sí $\square \quad$ NO $\square$

Si la pregunta 19 es afirmativa responda la pregunta 20.

20. En el caso de las subvenciones recibidas: ¿Cuál es el criterio de reconocimiento de los Activos no monetarios? En caso de haber utilizado más de un criterio, presente un detalle mencionado en qué casos utiliza cada criterio y proporcione los valores de los activos recibidos. 